\title{
Colonna Curva
}

\section{A case study on curved folding for the production of architectural components}

\author{
Marco Hemmerling \\ Cologne University of Applied Sciences, Germany \\ marco.hemmerling@th-koeln.de \\ Alessio Mazzucchi \\ Politecnico di Milano, Italy \\ mazzucchi.alessio@outlook.com
}

\begin{abstract}
The research presented in the paper focuses on computational folding. Besides the well-known straight folded structures, like the classical Miura fold, curved folding opens up more complex spatial configurations and delivers at the same time more performative structural effects, as the bended surfaces resulting from the curved crease folding enhance the overall-stiffness of the structure. Against this background the paper discusses the potential of curved folding techniques for the design and fabrication of architectural components. The findings are illustrated in a case study that documents the prototypical realization of a curved column in scale 1:1.
\end{abstract}

Keywords: curved folding; developable surfaces; deployable structures; parametric design; digital fabrication.

\section{Introduction}

The development of computational tools since the 1970s has given rise to new approaches towards geometric complexity. The research presented in this paper focuses on the field of computational folding, which has reshaped the traditional origami art into a branch of mathematical studies. Developable surfaces appear naturally when spatial objects are formed from planar sheets of material without stretching or tearing. The striking elegance of models folded from paper appear not only visually interesting, they inherit moreover positive structural effects and allow for the creation of multiple pieces from one folded sheet, which may save material, fabrication time and building costs. Hence, the ability to create a structural form from thin, flat materials has made folded structures an ideal candidate for lightweight deployable structures in architecture and engineering.

Origami (from ori meaning "folding", and kami meaning "paper") has evolved over the centuries from a traditional Japanese folk art to a design strategy for contemporary architecture and products. It was the rise of the art of Origami that revealed a whole new world of beauty and complex geometries which are not only sculptures made from just one piece of paper but a whole dynamic system of great complexity and logic. Many of those complexities remained a mystery and were considered an artistic virtue rather than a mathematical phenomenon. It was seen as more of an art than a true system and indeed it took great craftsmanship and expertise to create Origami art. In traditional origami, the folding sequences and titles are passed down as something anonymous, not as something made up by a specific person. Modern origami, which started in the 20th century, is based on a completely different paradigm. The folding sequences of modern origami are regarded as "models" "designed" by "origami creators." One of the most influential origami artists and theorists of our time is the US-American physicist Robert J. Lang (2011). He has not only contributed many publications on folding strategies but also made great advances in developing real-world applications of origami to engineering problems. Furthermore, the experimental work of origami artist Ron Resch (www.ronresch.org) and the research by computer scientist David A. Huffman (Wertheim 2004) in the 1970s laid the foundation for contemporary folding techniques.

The folding mechanism of a rigid material may seem as trivial as folding a sheet of paper. A simple crease along a line or a curve which dictates a determined movement for both faces. The basic origami folds include valley and mountain folds, pleats, reverse folds, squash folds, and sinks. The number of basic folds is small, but they can be combined in a wide variety of ways to make intricate designs. The Miura fold is one type of a flat fold rigid origami model, which consists of a combination of basic folds and has strong resistance in one direction and weak resistance in the other. One of its appeals is that it allows one to take a bendable piece of paper and from it create seemingly rigid surfaces. The reason for this is the small face area which the paper is split into. The smaller the face area, the more rigid the material will be as the ratio between the material thickness and the surface decreases.

\section{Curved Folding}

Since the art of paper folding is strongly related to geometric principles origami has received a considerable number of mathematical studies. Besides the well-known straight folded structures, like the classical Miura fold and other conventional tessellation techniques, curved folding opens up more spatial options and delivers at the same time more performative structural effects, as the bended (single-curved) surfaces 
resulting from the curved crease folding enhance the overallstiffness of the structure. However, curved folding addresses complex mathematical topics such as differential geometry and optimization algorithms that require an in-depth understanding of the underlying principles (Kilian et al., 2008). In order to reduce the complexity, we defined a clear framework to control (design) and produce (fabrication) the curved crease folding structure, the following conditions where defined for the further process:

\section{Developable surfaces}

Developable surfaces are surfaces, which can be unfolded into the plane while preserving the length of all curves on the surface. They are composed of planar patches and patches of ruled surfaces with the special property that all points of a ruling have the same tangent plane. There exist various methods to unroll a 3D-geometry, based on curved folding, which are composed by single-curved surfaces into planar elements. (Pottmann et. al, 2007, Glaeser et al., 2007, Shatz et al., 2006). However, to ensure the unfoldment into a single developable sheet without the need to cut or glue the various parts is still quiet challenging. Hence, to use the full potential of the origami folding principles the perquisite was set to fold the resulting shape from a single sheet of thin planar material (e.g. paper). Mathematically, an origami surface is a surface that consists of multiple developable patches and can be unfolded into a plane without cutting along creases.

\section{Mirror reflection}

The creation of the surface is based on planar curved folds and the mirror reflection. The key idea is to use mirror reflection through an implicitly defined plane (figure 1). Beginning from a simple initial single curved surface, more complex surfaces with multiple curved folds can be defined by continuously adding folds.
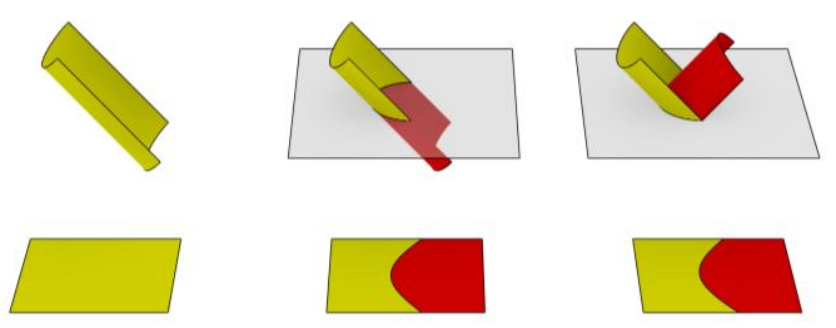

Figure 1: construction principle of a curved-folded geometry, based on the mirror reflection. The yellow single-curved surface is divided by a cutting plane that serves as well as the mirror plane (sequence above, from left to right). The unrolled surface shows the curved folding crease, between the yellow and red patches (sequence below).

Referring to the research by David A. Huffman (1976), the pair of generating lines at any point on the fold makes equal angles with that plane, when a curved fold is contained in a single plane. This means that the plane acts as a reflection plane that reflects the tangent plane on one side to the tangent plane on the other side (Mitani et al., 2011). Hence, we can state that a shape generated by applying mirror reflection to part of an origami surface is also an origami surface. By taking into account that the various planes do not intersect each other, complicated origami surface with planar curved folds can be obtained by applying multiple reflections to a simple origami surface.

\section{Case study - Colonna Curva}

Based on the assumptions described above, a parametric model was developed in Rhinoceros/Grasshopper that makes possible the design of various curved folded columns and generates automatically the unrolled surface with the creased folding pattern for fabrication.

\section{Design process}

In a first design step the principles of the mirror plane construction, as described in the last section, were implemented in a Grasshopper logic to generate a parametric tool for the further design process. The definition of a planar input curve, defined in Rhinoceros, served both as a starting point for the extrusion of the first developable surface and as a design manipulator for the system. By changing the curvature of the input curve the designer can generate variations of the curved folding. A mirror plane (also defined in Rhinoceros) reflects the extruded surface onto a consecutive surface. By changing the position and/or the angle of the mirror plane the resulting curved folding can also be manipulated by the designer (figure 2). The resulting patches of the curved folding surface are oriented in opposite directions $($ red $=$ convex, yellow $=$ concave $)$.

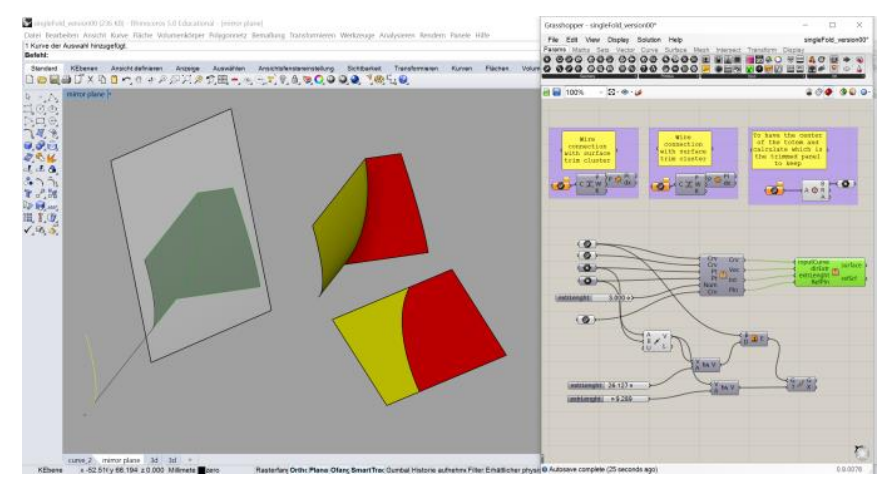

Figure 2: programming of the geometry in Rhinoceros/Grasshopper showing the input parameters (yellow line and grey mirror plane on the left) as well as the resulting curved-folded geometry (yellow and red patches of the resulting surface on the right, both in 3D and as an unrolled pattern).

The extension of the principle towards a more complex structure was then carried out with the aim to create a volumetric geometry that could serve as a building component in scale 1:1. In order to obtain a closed volume multiple mirror planes needed to be defined on a polygonal grid, such that the result follows the sequence of convex and concave patches. Hence, a hexagonal grid (as it is based on even numbers) was chosen as a starting point for the further design process. The previous Grasshopper logic was extended based on these assumptions. In order to close the volume, five mirror planes were organized around the center of a circle, such that the planar input curve was reflected continuously along the mirror planes until the final patch 
intersects the first patch of the curved folding surface (figure 3).

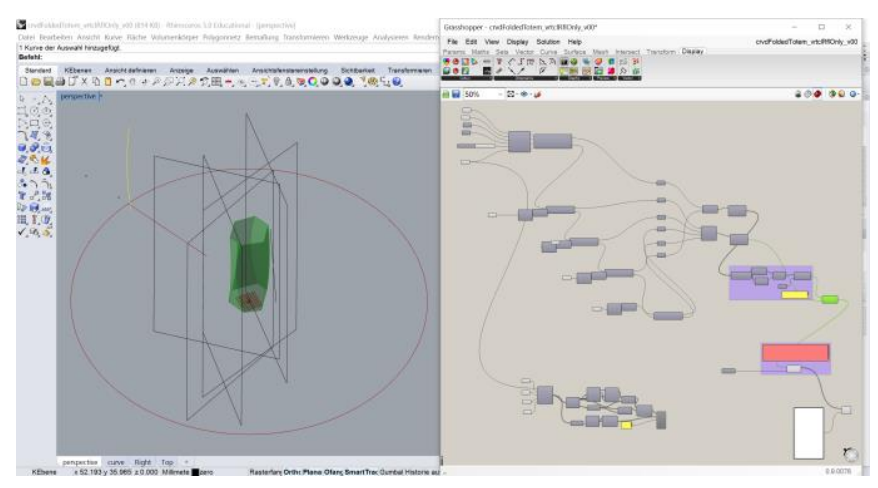

Figure 3: programming of the column geometry: The reflection of the extruded yellow input curve on the five black mirror planes result in a hexagonal curved folding volume with consecutive convex and concave patches.

Based on this parametric model variations of the design were developed by changing the geometry of the planar input curve (with different curvature orientations) and/or by changing the position and/or angle of the mirror planes. The resulting developable surfaces were finally unrolled to proof the concept of the origami surfaces, i.e. the unfoldment into a single developable sheet (figure 4).

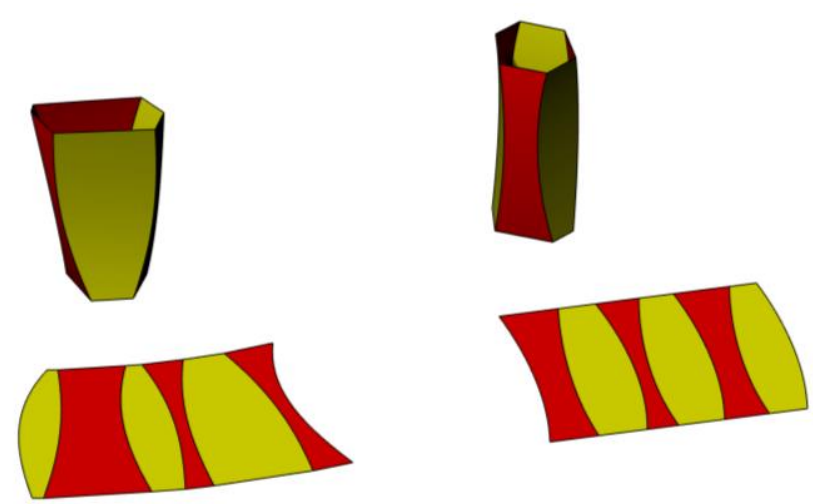

Figure 4: column variations and unrolled surfaces to a single sheet, both showing convex (red) and concave (yellow) patches.

\section{Fabrication process}

The first physical prototypes, which were carried out in paper and cardboard, verified the following assumptions:

- Origami surface (unfoldment into a single sheet, therefore also a fast assembly).

- Enhanced structural performance through curved folding (high stiffness of the model, especially through the convex/concave set-up).

Having experimented with foldability of different materials, the assumption was that a material should be used that has rigid properties on the one hand yet still be able to fold manually like a folded paper. Various materials were taken into account, ranging from plastics to sheet metal.

One of the most common techniques of plate folding is sheet metal folding. It is used heavily in industry and has many applications, mainly in profiling, flange connectors and machine construction. Due to the heavy pressure applied on both ends, the bend creates a plastic deformation with the fold angle, even after folding not needing any re-enforcing. Technically, the material is stressed beyond its yield strength but below its ultimate tensile strength. There is little change to the materials surface area. Bending generally refers to deformation about one axis only.

Another relatively new method of folding rigid materials is crease folding, which has been popularized with the invention of the composite boards in the late 70's. Though it could also be done with pure metal sheets, this method has gained popularity with ACM boards for the easiness of the folding process that can be done by hand thanks to the thin soft aluminum layers on which it is folded. In this method, the bend radius may be minimized to around $0.2 \mathrm{~mm}$. This allows the designer to neglect it and use it as a workable tolerance. The action of creasing a panel consists of cutting out core material along the line of the fold, leaving a very small amount of material (mostly aluminium) which is bent in the folding process. The section of the creased panel will depend on the revolving groove being used for engraving and its height from the zero $z$ axis (figure 5).

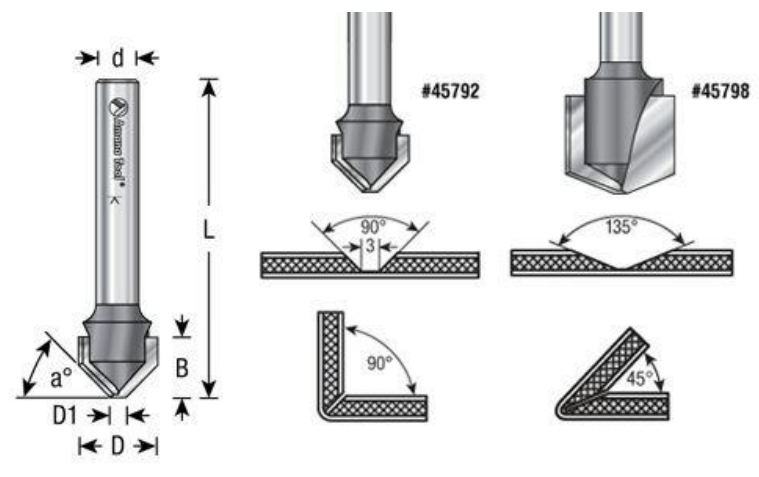

Figure 5: various types of grooves result in different folding angles and bending radii.

Hence, the result of the material research yielded that for this specific case, a composite aluminum board seemed to be the most promising approach. The properties of such material allow it to be creased along the fold lines, which creates an easily bendable fold while maintaining the surface rigidity. The fabrication method was applied to two 1:1 scale prototypes fabricated from Aluminum Composite Material (ACM) boards, which were creased by CNC-milling in the further process. Before the start of the fabrication, however, some considerations had to be taken regarding the manufacturing. The concept of the computational column folding is based on mathematical discrete surfaces, in other words, the resulting geometry has no thickness. While working with paper models this does not appear to be a problem as the material is very thin and also flexible enough to allow for tolerances. But, if we move to the scale of building components the material thickness matters. The main challenge is to avoid the material intersection when the 
panel is folded to its desired shape. While a mathematical model shows whether the faces are intersecting or not, it is not possible to retrieve the thickness intersection without modelling it. Other parameters to be taken into account are the bending angle which the material allows (elastic vs. plastic deformation) and the fatigue threshold as well as the processing of the material and the tooling (different engraving bits create different fold angles and bend radii).

The first prototype of a curved folding surface with a single crease was CNC-milled from a $2 \mathrm{~mm}$ ACM board of an overall dimension of $1.00 \mathrm{~m} \times 1.50 \mathrm{~m}$, using a $90^{\circ}$ milling bit. The folded structure was easy to bend and the resulting convex and concave patches of the surface showed a significant stiffness. However, the opposite sides of the origami surface were loose ends, such that the structure bended back due to the elasticity of the material and the tension of the curved patches of the surface.

The dimensions of the two final curved columns was chosen by the maximum available panel size $(2.00 \times 3.50 \mathrm{~m})$, with a material thickness of $3 \mathrm{~mm}$. As the smallest angle between the curved patches was slightly more than $90^{\circ}$ (thanks to the hexagonal geometry) we chose for a $90^{\circ}$ milling-bit to avoid material intersection, as described before. The CNC-milling process was fast, as the overall length of the crease lines was quite small in relation to the panel size (figure 6).
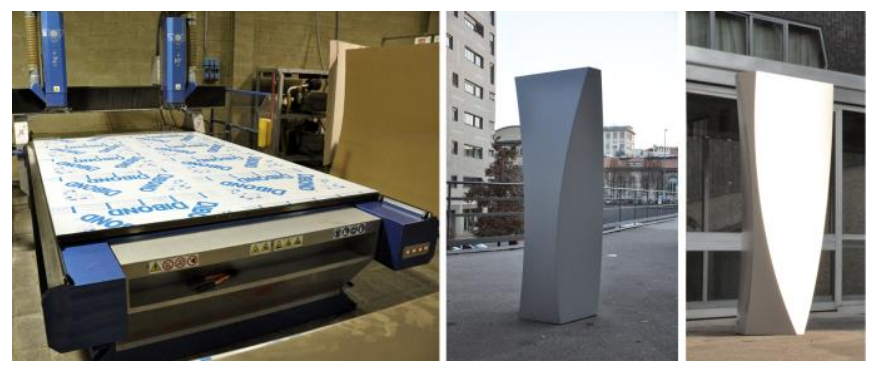

Figure 6: CNC-milling of the ACM boards (left) and resulting columns after assembly (right).

\section{Conclusion and Outlook}

The columns (approx. diameter: $60 \mathrm{~cm}$, height: $200 \mathrm{~cm}$ ) showed a significant overall stiffness, with a material thickness of only $3 \mathrm{~mm}$. The fabrication and assembly was fast and the material didn't buckle or show any fatigue from the bending. The columns are extremely light, while maintaining the shape also under pressure and tension. In addition to the fabrication of architectural components, like façade elements, the principle could potentially also be applied to complex casting molds of concrete columns or other building parts. A possible extension for the fabrication of the components can be found in the approach of RoboFold founder Greogry Epps, who also carried out the realization of the ARUM installation by Zaha Hadid for the Venice Biennale in 2012.

\section{Acknowledgments}

The research project was carried out at the Politecnico di Milano and supported by $3 \mathrm{~A}$ Composites (material) and Pigomma SpA (CNC-processing).

\section{References}

Glaeser, G., Gruber, F. (2007). Developable surfaces in contemporary architecture. J. of Math. and the Arts 1, 1-15.

Huffman, D. A. (1976). Curvature and creases: a primer on paper. IEEE Trans. Computers C-25, 1010-1019.

Lang, R.J. (2011). Origami Design Secrets: Mathematical Methods for an Ancient Art, Taylor \& Francis Ltd.

Mitani, J., Igarashi, T. (2011). Interactive Design of Planar Curved Folding by Reflection. Pacific Graphics.

Pottmann, H., Asperl, A., Hofer, M. Kilian, A. (2007). Architectural Geometry. Bentley Institute Press.

Shatz I., Tal A., Leifman G. (2006). Paper craft models from meshes. Vis. Comput. 22, 825-834.

Wertheim M (2004). Cones, curves, shells, towers: He made paper jump to life. The New York Times.

Ron Resch. Retrieved from http://www.ronresch.org

Rhinoceros. Retrieved from http://www.rhino3d.com

Grasshopper. Retrieved from www.http://grasshopper3d.com

Processing of ACM boards (figure 5). Retrieved form:

http://media.alucobond.com/pdf/alucobond/processing/alucobondproc essingen.pdf

RoboFold, Gregory Epps. Retrieved from http://www.robofold.com

ARUM installation by Zahah Hadid Architects. Retrieved from http://www.zaha-hadid.com/design/contribution-to-2012-venicebiennale-theme-\%E2\%80\%98common-ground\%E2\%80\%99/ 